\title{
Reflexões acerca do Ensino de Pediatria no Século XXI: o Cenário Brasileiro
}

\author{
Reflections on Teaching Pediatrics in $21^{\text {st }}$ \\ Century Brazil
}

\author{
Andréia de Santana Silva Moreiral \\ Alexandra Prufer Queiroz Campos Araújo ${ }^{I I}$ \\ Márcia Gonçalves Ribeiro II \\ Rodrigo Siqueira-BatistaIII
}

\section{PALAVRAS-CHAVE \\ - Ensino; \\ - Pediatria; \\ - Educação Médica.}

\section{KEYWORDS}

- Teaching;

- Pediatrics;

- Medical Education.
Reencaminhado em: 20/11/2014

Aprovado em: 18/04/2015

REVISTA BRASILEIRA DE EDUCAÇÃO MÉDICA

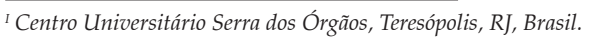

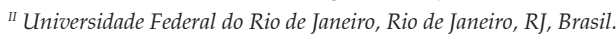

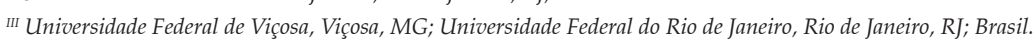




\section{INTRODUÇÃO}

O ensino de Medicina vem sendo discutido, amiúde, desde os primórdios do século XX, delimitando-se a publicação do Relatório Flexner, em 1910, como salutar ponto de partida nesse movimento ${ }^{1,2}$. Com base em interpretações desse documento, passou-se à práxis de um ensino médico centrado na doença considerada um processo natural, biológico, muitas vezes $a$-histórico -, na sólida formação científica (ciclo básico) e na eleição do hospital como lócus privilegiado do processo ensino-aprendizagem. De fato, como a formação laboratorial acontecia no ciclo básico e a formação clínica em hospitais, estes se transformaram na principal instituição de transmissão de conhecimento médico durante todo o século $X^{1,3}$. O social, o coletivo e o ambiental não contavam - ou possuíam importância secundária - para a formação do discente, na medida em que não eram percebidos como parte do processo de saúde-doença ${ }^{4}$.

A necessidade de mudanças tornou-se evidente na segunda metade do século passado, mormente nos anos de 1980 e 1990, quando surgiram questionamentos sobre o perfil do profissional formado - destacando-se a preocupação com a especialização precoce ${ }^{1}$. Nesse contexto, surge a Lei de Diretrizes e Bases da Educação Nacional (LDBEN) no cenário da educação superior, que define entre suas finalidades o estímulo ao conhecimento dos problemas do mundo atual (nacional e regional) e a prestação de serviço especializado à sociedade, estabelecendo uma relação de reciprocidade aparelho formador/coletividade ${ }^{5,6}$

Após várias discussões, em 2001, o Ministério da Educação homologa as Diretrizes Curriculares Nacionais do Curso de Graduação em Medicina (DCN) ${ }^{2,6}$. O vetor do desenvolvimento curricular passa a se orientar para as necessidades de saúde da população, preconizando a interação entre o ensino, o serviço e comunidade ${ }^{7}$. Com a homologação das DCN, as instituições formadoras são convidadas a mudar suas práticas pedagógicas de modo a estimular o corpo discente - por intermédio da mediação docente - a tecer novas redes de conhecimento em prol da aquisição de competências e habilidades essenciais à prática profissional e ao trabalho em saúde ${ }^{8}$.

Considerando-se, ainda, que a graduação dura somente alguns anos - ao passo que a atividade profissional pode permanecer por décadas - e que os conhecimentos vão se transformando velozmente, torna-se essencial pensar em metodologias para uma prática educacional libertadora, na formação de um profissional ativo e apto a aprender a aprender ${ }^{5,7}$. Muitas escolas médicas vêm lançando mão de estruturas curriculares baseadas nas metodologias ativas de ensino-aprendizagem, dentre as quais se destaca a aprendizagem baseada em problemas $(\mathrm{ABP})$, nas quais o aluno se torna o centro do aprendizado ${ }^{5,7}$.
As DCN propõem que o médico deve ter uma formação generalista, humanista, crítica e reflexiva e deve atuar como promotor da saúde integral do ser humano ${ }^{6}$. A Pediatria é a área da Medicina em que a promoção da saúde integral tem a maior importância, pois a criança é o futuro adulto.

Pedro de Alcântara ${ }^{9}$ descreveu a Pediatria como o campo da Medicina que atende aos problemas da criança, isto é, do ser humano em seu período de desenvolvimento - da fecundação à puberdade. Referiu que tal campo de saber-fazer se organiza mediante duas formas por assim dizer "isômeras" de atividade: a Puericultura, que cuida da manutenção das condições de normalidade, e a Clínica Pediátrica ou Pediatria Curativa, que cuida de sua restauração quando alterada. Segundo o autor, são como irmãs xifópagas, uma complementando a outra e dela necessitando 9 . Por esta definição, a Pediatria seria um domínio peculiar da Medicina que assiste o ser humano como um "todo" durante determinado momento do ciclo de sua vida.

Tal definição data do século XX. Desta feita, cabe a indagação plenamente atual: $O$ que se espera do médico geral em relação à Pediatria no século XXI? E, como questão dela derivada: Mudanças nas diretrizes para o ensino de Pediatria são necessárias a partir das DCN? Este artigo objetiva discutir tais questões, tendo como fulcro as DCN e as Diretrizes para o Ensino de Pediatria propostas por Eduardo Marcondes em 1993.

\section{MÉTODOS}

Trata-se de um ensaio reflexivo elaborado com base em dois documentos: Diretrizes Curriculares Nacionais do Curso de Graduação em Medicina (DCN), de novembro de 20016; ; e Diretrizes para o Ensino de Pediatria (DEP) propostas por Eduardo Marcondes e publicado no Jornal de Pediatria em $1993^{10}$.

Procedeu-se à leitura dos dois textos e à análise de ambos, em conjunto, a partir de referências bibliográficas selecionadas pelos autores.

\section{DISCUSSÃO}

$\mathrm{O}$ artigo $4^{\circ}$, parágrafo I, das DCN - no que tange a atenção à saúde - refere que o médico deve estar apto a desenvolver ações de prevenção, promoção, proteção e reabilitação da saúde, em nível tanto individual quanto coletivo. Nas DEP, observa-se, na sua primeira percepção, que a criança deve ser avaliada como um todo, reagindo, adoecendo e necessitando de assistência como tal ${ }^{10}$. Em relação à assistência, é aplicado o termo "Puericultura" - que significa criação da criança (puer = criança; cultura $=$ criação). Segundo Martagão Gesteira ${ }^{11}$, esta é a

[...] parte da Medicina que se ocupa em cultivar a vida e a saúde das crianças, esforçando-se para que cheguem ao mun- 
do sadias e fortes e se desenvolvam normalmente, amparando-as e defendendo-as contra múltiplos perigos que as ameaçam em consequência da ação maléfica dos fatores ambientais ou sociais. (p.21)

Este termo foi usado pela primeira vez em 1762 por Jaques Ballexserd e foi revivido por Caron em 1865. Ele vislumbrou, então, dois pilares magnos para a Puericultura: a prevenção e a educação para a saúde em geral ${ }^{10,11}$.

Passados mais de 150 anos, tais pilares são atuais e propostos pelas DCN - por exemplo, no excerto "os profissionais de saúde, dentro do seu âmbito profissional, devem estar aptos a desenvolver ações de prevenção, promoção, proteção e reabilitação da saúde, tanto em nível individual quanto coletivo" -, devendo, portanto, ser mantidos quando pensamos no ensino de Pediatria no século XXI.

A segunda percepção de Marcondes volta a referir a promoção da saúde, a prevenção de agravos e a educação da criança e da família. A higiene alimentar e a orientação na prática de exercícios seriam promoção de saúde, enquanto as imunizações e a prevenção de acidentes seriam prevenção de $\operatorname{agravos}^{10}$. Tal percepção está incluída na Puericultura e recomendada no mesmo artigo e parágrafo das DCN. Já a educação da criança e da família proposta nesta percepção está amparada, nos dias atuais, no artigo $5^{\circ}$, parágrafo IV das DCN:

\section{[...] informar e educar seus pacientes, familiares e comuni- dade em relação á promoção de saúde, prevenção, tratamento e reabilitação das doenças, usando técnicas apropriadas de comunicação. (p.2)}

A terceira percepção de Marcondes se refere ao ambiente onde a criança vive, que inclui os elementos físicos/ecológicos e psíquicos/sociais. Esse autor atenta para o fato de que a doença é resultante da inexistência, ineficiência ou insuficiência de adaptação do homem às características ou alterações do ambiente. As DCN propõem como competências e habilidades específicas, em seu artigo 5º, parágrafo I, a promoção de estilos de vida saudáveis, conciliando as necessidades tanto dos usuários do sistema de saúde quanto da comunidade, colocando o médico como agente de transformação social. No parágrafo VI do mesmo artigo, observa-se, ainda, a necessidade de o médico ter como competência o domínio dos conhecimentos científicos básicos da natureza biopsicossocioambiental da prática médica. $\mathrm{O}$ ambiente e a sua relação com o processo saúde-doença do cidadão são colocados como conteúdo essencial, nos dias de hoje, no artigo $6^{\circ}$ das DCN, cabendo lugar destacado para os mesmos no âmbito da educação médica ${ }^{6,10,12,13}$.
A obtenção de dados - que reflete a arte da Medicina de realizar a anamnese, a construção da história clínica e a técnica do exame físico - está contemplada na quarta percepção de Marcondes e nas DCN, no artigo $5^{\circ}$, que concerne às competências e habilidades específicas do médico, e no artigo $6^{\circ}$, que coloca como conteúdo essencial a compreensão e o domínio da propedêutica médica ${ }^{6,10}$

A quinta percepção de Marcondes trata do acompanhamento do crescimento e do desenvolvimento infantil. São fenômenos distintos, mas intimamente relacionados. Enquanto crescimento significa aumento somático - como um todo ou em partes - e pode ser medido em centímetros e quilos, desenvolvimento significa aumento da capacidade do indivíduo na realização de funções cada vez mais complexas. Desta forma, não basta medir e pesar crianças; é preciso incluí-las em gráficos de crescimento a fim de obter a sua "rota de crescimento". Há necessidade de conhecimentos acerca do desenvolvimento infantil - do ponto de vista neuromotor e psicológico - para que se possa acompanhar e identificar precocemente os seus desvios 9 .

A publicação original de Marcondes aponta como parâmetro básico de desenvolvimento a atividade da criança, ou seja, seu desenvolvimento motor. No século atual, é essencial conhecer o desenvolvimento infantil nos três domínios principais: motor, linguístico e cognitivo. O reconhecimento de desvios do desenvolvimento é de extrema importância, pois o tratamento e a reabilitação farão diferença no futuro desses indivíduos. Tal constatação torna-se particularmente relevante ao se considerar que, ao longo dos últimos anos, houve diferenças na morbidade infantil, e situações que interferem no desenvolvimento são cada vez mais frequentes. Pode-se citar como exemplo o avanço tecnológico, que permite que bebês prematuros - antes condenados ao desenlace fatal - sobrevivam atualmente, mas com uma morbidade maior, tipificada, muitas vezes, por grandes desvios em seu desenvolvimento ${ }^{14}$. $\mathrm{O}$ aumento da incidência de autismo infantil e de situações crônicas de morbidade cognitiva - como o transtorno do déficit de atenção e hiperatividade (TDAH) e o retardo mental ${ }^{14}$ - é uma realidade no mundo atual, não podendo ser negligenciado, quanto ao reconhecimento, pelo profissional médico.

$\mathrm{O}$ artigo $5^{\circ}$ das DCN, que contempla as competências e habilidades específicas do médico, aponta, em seus parágrafos VII, VIII e XIII, as recomendações necessárias para acompanhar o crescimento e o desenvolvimento infantil e seus desvios, tais como a competência e habilidade de diagnosticar e tratar as principais doenças do ser humano, tendo em vista o potencial mórbido e a prevalência de cada uma, reconhecer e encaminhar adequadamente pacientes que fujam ao alcan- 
ce de sua formação geral, bem como o papel de promoção da saúde, prevenção de doenças e reabilitação do indivíduo.

A sexta percepção de Marcondes trata da primazia da nutrição na infância, sendo papel do médico a orientação quanto à alimentação, o controle do estado nutricional e a averiguação dos seus desvios, além do reconhecimento dos recursos para correção destes problemas. Vale lembrar o papel do médico como incentivador e promotor do aleitamento materno. Quanto a esta percepção, observam-se mudanças nas últimas duas décadas, com aumento substancial e crescente, no mundo todo, da obesidade infantil e de suas consequências ${ }^{14}$. As competências e habilidades necessárias para acompanhar e tratar a nutrição infantil são as mesmas para acompanhar o crescimento e o desenvolvimento.

A sétima e última percepção versa sobre a prescrição pediátrica, afirmando que esta deve ser clara e legível - como claramente enunciado no atual Código de Ética Médica - e que consta de quatro itens: (i) alimentação, (ii) imunização, (iii) medicação e (iv) orientação. Tal percepção vai ao encontro do artigo $4^{\circ}$ das DCN no parágrafo III, quando trata da competência e habilidade do médico em relação a comunicação.

No estudo realizado por Veiga e Batista ${ }^{15}$, que buscou descrever o ensino de Pediatria nas escolas médicas no Estado do Rio de Janeiro, foi observado que os temas mais presentes nos currículos de graduação em Pediatria são os relacionados à introdução à Pediatria, com ênfase em anamnese e exame físico, ações básicas de saúde (em especial, o aleitamento materno), prevenção de acidentes na infância, acompanhamento do crescimento e desenvolvimento, atendimento ao recém-nascido normal, imunizações e controle das infecções respiratórias e doenças diarreicas. Tal observação corrobora as recomendações feitas por Marcondes e contempladas nas DCN. Em contrapartida, temas referentes aos aspectos psicomotor e afetivo, como habilidades de comunicação na relação pediatra-criança-família, não são contemplados em todas as instituições pesquisadas, o que vai em direção oposta à formação médica pretendida nos dias atuais ${ }^{15}$.

\section{CONSIDERAÇÕES FINAIS}

De todas as áreas de atuação da Medicina, a Pediatria foi a que se antecipou na aplicação prática das considerações da LDBEN e das DCN, incorporando no contexto da Puericultura os aspectos do cuidado para além do biológico, englobando o social, o coletivo e o ambiental. Embora a Pediatria seja o duplo marco inicial, do ciclo da vida e da implantação de prática holística, as atualizações do currículo médico devem perpassar o ciclo completo de vida do ser humano, haja vista a relação entre os extremos deste ciclo ${ }^{16}$.
Marcondes, mediante suas percepções, propõe as Diretrizes para o Ensino de Pediatria - as quais parecem se manter extremamente atuais no contexto brasileiro do século XXI -, atentando apenas para diferenças de mortalidade e morbidade. As DEP poderiam ser resumidas nos seguintes tópicos:

(a) Entendimento da criança como unidade biopsicossocial e ecodependente;

(b) Promoção de saúde, prevenção de agravos e educação familiar: Puericultura;

(c) Monitorização do crescimento, desenvolvimento e nutrição infantil, e reconhecimento dos seus desvios;

(d) Elaboração de diagnóstico e prescrição de tratamento, a partir da capacidade de obter dados por meio da anamnese e do exame físico e conhecimento das doenças prevalentes neste ciclo da vida, em níveis de unidade básica de saúde, consultório de emergência, enfermaria geral e berçário;

(e) Conhecimento teórico-prático sobre estudos de mortalidade infantil nacional e regional, valor da equipe de saúde na atenção à criança, educação em saúde, capacitação para executar procedimentos diagnósticos e terapêuticos fundamentais.

Segundo Marcondes, tais Diretrizes seriam adequadas tanto ao ensino de graduação como à especialização, diferenciando-se apenas a carga horária necessária a cada um. Levando-se em conta esta recomendação de Marcondes, é importante citar que a Sociedade Brasileira de Pediatria (SBP) participa da discussão para construir um modelo de currículo global para o ensino de Pediatria que passaria a ser referência no Brasil. Nesse novo currículo, a saúde mental passa a ser conteúdo explícito e obrigatório; a adolescência tem sua carga horária ampliada; as doenças crônicas são incluídas obrigatoriamente; treinamentos em neonatologia são ampliados; a Puericultura é aprofundada, com ênfase na influência do meio ambiente no crescimento e no desenvolvimento com vistas à saúde do adulto $^{17}$.

Observando as recomendações do novo currículo global para o ensino de Pediatria, as DEP, respeitando pequenas modificações abordadas neste artigo, não devem ser negligenciadas nos currículos médicos atuais, tendo em vista a formação do médico generalista, humanista, crítico e reflexivo que se pretende atualmente.

\section{REFERÊNCIAS}

1. Pagliosa LF, Da Ros MA. O Relatório Flexner: para o bem e para o mal. Revista Brasileira de Educação Médica. 2008; 32(4):492-499.

2. Maranhão EA, Gomes AP, Siqueira-Batista R. O que mudou na educação médica a partir das Diretrizes Curriculares Nacionais: sob os olhares do Jano de Duas Faces. In: 
Streit DS, Barbosa Neto F, Lampert JB, Lemos JMC, Batista NA. 10 anos de Diretrizes Curriculares Nacionais. Rio de Janeiro: ABEM; 2012. p. 59-91.

3. Filho NA. Reconhecer Flexner: inquérito sobre a produção de mitos na educação médica no Brasil contemporâneo. Cadernos de Saúde Pública. Rio de Janeiro. 2010; 26(12):2234-2249.

4. Gomes AP, Rego S. Transformação da educação médica: é possível formar um novo médico a partir de mudanças no método de ensino-aprendizagem? Revista Brasileira de Educação Médica (Impresso). 2011; 35:557-566.

5. Mitre SM, Siqueira-Batista R, Girardi-de-Mendonça JM, Pinto NMM, Meirelles CAB, Porto CP, et al. Metodologias ativas de ensino-aprendizagem na formação professional em saúde: debates atuais. Ciência e Saúde Coletiva. 2008; 13(Sup 2):2133-2144.

6. Brasil. Ministério da Educação. Conselho Nacional de Educação. Câmara de Educação Superior. Diretrizes Curriculares Nacionais do Curso de Graduação em Medicina. Resolução CNE/CES 4/2001. Brasília: Ministério da Educação; 2001.

7. Siqueira-Batista R, Rôças G, Gomes AP, Albuquerque VS, Araújo F, Messeder JC. Ecologia na formação do profissional de saúde: promoção do exercício da cidadania e reflexão crítica comprometida com a existência. Revista Brasileira de Educação Médica (Impresso) 2009; 33:271-275.

8. Siqueira-Batista R, Gomes AP, Albuquerque VS, Cavalcanti FOL, Cotta RMM. Educação e competências para o SUS: é possível pensar alternativas à(s) lógica(s) do capitalismo tardio? Ciência e Saúde Coletiva (Impresso). 2013; 18:159170.

9. Alcantara P. Introdução ao estudo da Pediatria. In: Marcondes E. Pediatria Básica. 8.ed. São Paulo: Sarvier; 1992. p 3-9.

10. Marcondes E. Diretrizes para o ensino de Pediatria. Jornal de Pediatria.1993; 69(6):349-352.
11. Crespin J. Puericultura: Ciência, Arte e Amor. 1.ed. São Paulo: Fundação Byk; 1992.

12. Siqueira-Batista R, Rôças G. Alfabetização ecológica. Revista Brasileira de Educação Médica 2009; 33:123-125.

13. Siqueira-Batista R, Siqueira-Batista R. Os anéis da serpente: a aprendizagem baseada em problemas e as sociedades de controle. Ciência e Saúde Coletiva. 2009; 14(4):1183-1192.

14. Staton B, Behrman RE. O Campo da Pediatria. In: Kliegman RM, Behrman RE, Jenson HB, Staton BF, et al. Nelson, Tratado de Pediatria. 18.ed. Rio de Janeiro: Elsevier; 2009. p.1-12.

15. Veiga EQO, Batista NA. O ensino de pediatria no nível de graduação nas escolas de medicina do estado do Rio de Janeiro. Jornal de Pediatria. 2006; 82(2):115-120.

16. Paixão CM, Araújo APQC. Frailty and vulnerability: Are the two terms equivalent in paediatrics and geriatrics? European Geriatric Medicine. 2010;1:166-169.

17. Sociedade Brasileira de Pediatria. Disponível em: www. sbp.com.br. [Acesso em: 01 out. 2014].

\section{CONTRIBUIÇÃO DOS AUTORES}

Os autores participaram igualmente de todas as etapas de elaboração desse artigo.

\section{CONFLITO DE INTERESSES}

Não há conflito de interesses.

\section{ENDEREÇO PARA CORRESPONDÊNCIA}

Andréia de Santana Silva Moreira

Instituto de Pediatria e Puericultura Martagão Gesteira IPPMG - UFRJ - Ambulatório de Neurologia Infantil Rua: Bruno Lobo nํㅗ. 50 .

Cidade Universitária - Rio de Janeiro

CEP: 21941-912 RJ

E-mail: andreiassm3@hotmail.com 